\title{
HISTOPATHOLOGICAL BASED RETROSPECTIVE STUDY OF ORAL KERATOTIC WHITE LESIONS IN MANIPAL HEALTH SYSTEMS-HOSPITAL.
}

\author{
P. Gurung*, J.B. Sherchan** and K. Pai*** \\ *Department of Pathology, Kathmandu Medical College, Sinamangal. \\ **Kathmandu University School of Medical Science, Dhulikhel. \\ ***Department of Pathology, Kasturba Medical College, Manipal.
}

\begin{abstract}
Oral keratotic white lesions are a common problem that is encountered on routine clinical examination. Clinical appearance of the lesion may belie the true nature of the lesion. So a biopsy followed by histopathological diagnosis is the gold standard in evaluating these lesions for malignant potential or dysplasias. Objective: The aim of the present study is to evaluate the clinicopathologic findings of oral keratotic lesions. Materials and Methods: Oral biopsies of 61 cases of oral keratotic white lesions during the period from January 2006 to July 2009 were studied retrospectively at the Department of Pathology, Kasturba Medical College, Manipal by microscopy. Clinical details and records were obtained from the medical records department. Results: In 61 cases of oral keratotic white lesions the age distribution ranged from 29 to 86 years. 66\% were males while $34 \%$ were females and most lesions occurred between 31 and 70 years. The buccal mucosa was the most common site of lesion in 35\% patients. Only 15 cases had some personal habits and 8/15 cases (53\%) showed dysplasia. 12/ 54 cases (22\%) of homogenous leukoplakia displayed dysplasia, of which 11 cases (92\%) showed mild dysplasia and 1 case (8\%) showed moderate dysplasia. Of the 4 cases of speckled leukoplakia, $75 \%$ showed dysplasia- mild, moderate and severe. $2 / 3$ cases (66\%) of verrucous leukoplakia displayed dysplasia- moderate and severe. There were 41 cases consistent with leukoplakia (67\%), of which 22\% showed epithelial hyperplasia and hyperkeratosis, 12 cases showed mild dysplasia, 3 cases moderate dysplasia and 2 cases showed severe dysplasia. Benign keratosis formed the largest group (35\%) among the 61 cases. Of the 61 cases 17 (27\%) showed dysplasia of which 19\% was mild dysplasia, 5\% was moderate dysplasia and 3\% was severe dysplasia. Most frequent histomorphological feature seen in this study was nuclear pleomorphism in 21/ 27 cases (78\%). There were 12 cases (71\%) of dysplasia in males and 5 cases (28\%) of dysplasia in females. There was 1 case of Progressive verrucous leukoplakia in an 86 year old female patient. There were 3 cases (5\%) each of oral lichen planus, lichenoid dysplasia and lichenoid keratosis in the present study. 6 cases (9\%) of candidiasis was seen among the 61 biopsies. In the study there was 1 case (2\%) each of verrucous carcinoma, squamous cell carcinoma and micro-invasive squamous cell carcinoma. Conclusions: Oral keratotic white lesions demonstrate a wide spectrum of histopathological features from benign lesions to dysplastic lesions to carcinoma in situ to invasive ones.
\end{abstract}

Key words: Histopathology; Retrospective; Oral keratotic white lesions.

\section{INTRODUCTION}

Oral keratotic lesions are frequently found during clinical examination. Some of these white lesions can be removed by scraping and are referred to as "Nonkeratotic" while some of the white lesions resist removal by scraping and are referred to as "Keratotic". The term leukoplakia is used to refer to these keratotic white patch or plaque on the oral mucosa. Leukoplakia is an exclusively clinical term and there is no histologic picture that is uniquely characteristic of this process. These lesions sometimes can have a definite cause like infection, systemic disease etc. Some of these oral white lesions may be associated with premalignant changes or even carcinoma, a fact that has been known for a long time. These lesions may precede the appearance of cancer by months or years, or they may be present together with the carcinoma when the patient is first seen. Some of the keratotic white lesions may be due to an increased keratinisation as a result of friction or repeated trauma or chronic irritation and may histologically show hyperkeratosis (e.g. frictional keratosis, traumatic keratosis). Other conditions like lichen Planus, hyperplastic candidiasis, etc. can appear clinically as oral keratotic white lesions. Clinically the white keratotic lesions due to varied etiology may have similar appearance, but have

Author for Correspondence: Dr. Pranita Gurung, Lecturer, Department of Pathology, Kathmandu Medical College, Sinamangal, Nepal.

Email: pranitagurung2009@gmail.com. 
a considerable degree of microscopic heterogeneity ranging from benign hyperkeratosis to varying degrees of epithelial dysplasia to frank invasive malignancy, and sometimes suggest a definite diagnosis like candidiasis, lichen planus. Biopsy followed by histopathological diagnosis is the gold standard in the evaluation of oral keratotic white lesions. Dysplasias are well known for their propensity for malignant transformation to the extent of 5-18\% ${ }^{1}$. Survival rates for oral cancer are very poor at approximately $50 \%$ survival overall, which may be due to late detection. Hence, the objective of the study was to evaluate the spectrum of histopathological findings in lesions presenting as oral keratotic white lesions and to determine the association with known risk factors.

\section{MATERIALS AND METHODS}

A histopathological based retrospective study was conducted in oral biopsies of 61 cases of oral keratotic white lesions during the period between January 2006 to July 2009 at the Department of Pathology, Manipal Health System, Hospital with the help of light microscopy.

The medical records of the patients were reviewed in detail according to the predefined criteria for the selection of cases such as oral keratotic white lesions excluding already diagnosed cases, patients diagnosed with carcinoma and non-keratotic white lesions.

\section{CLINICAL DATA}

Patient clinical data included in the study were sex, age at presentation, personal habits like betel usage, tobacco smoking, alcohol consumption, others and family history of oral cancer. Clinical examination included site of the lesion and morphology of the lesion whether lesion was homogenous, speckled, red and or erosive, verrucous or papillary.

\section{HISTOPATHOLOGICAL DATA}

The specimen consisted of excisional biopsy performed on small lesions, incisional biopsy performed on large lesions and multiple specimens from different areas. The tissue samples were fixed in 10\% neutral buffered formal saline. It was then processed in LEICA TP 1050 or LEICAASP 200S, embedded in paraffin and cut at a thickness of 5 microns and blocks and slides prepared. Slides were stained with routine Haematoxylin and Eosin stain and then studied by two Pathologists using light microscopy following the WHO 2005 criteria ${ }^{2}$. Whenever special stains like Periodic acid Schiff (PAS) were required, additional slides of the same were made. Results were then correlated with the clinical history of the patients which were obtained from the patient's files from the medical records department.

\section{RESULTS}

A total of 61 biopsies of cases from oral keratotic white lesions were studied. 40 (66\%) were male and 21 (34\%) were female patients in which average age was 50 in male and 54 in female.
The prevalence of oral keratotic white lesions, their clinical appearance and their correlation to histopathological findings was higher in males than females in the ratio of 2:1 and 27\% of the cases were diagnosed to have different grades of dysplasia as shown in Table 1.

\section{Site of lesions and grade of dysplasia:}

In the study four different sites of Leukoplakia were found with different grade of dysplasia in which 12 patients had mild, 3 moderate and two cases had severe

Table 1 Correlation of the grade of dysplasia with age and sex distribution

\begin{tabular}{ccccccccccc}
\hline \multicolumn{10}{c}{ Men } & \multicolumn{9}{c}{ Women } \\
\hline Age & Mild & Moderate & Severe & Total & Mild & Moderate & Severe & Total & Cases & Percent \\
\hline $20-30$ & - & - & - & - & - & - & - & - & - & - \\
$31-40$ & 1 & 1 & - & 2 & - & - & - & - & 2 & $3 \%$ \\
$41-50$ & 3 & 1 & 1 & 5 & 1 & - & - & 1 & 6 & $9 \%$ \\
$51-60$ & 1 & 1 & 1 & 3 & 2 & - & - & 2 & 5 & $8 \%$ \\
$61-70$ & 1 & - & - & 1 & 2 & - & - & 2 & 3 & $5 \%$ \\
$71-80$ & 1 & - & - & 1 & - & - & - & & 1 & $2 \%$ \\
Total & 7 & 3 & 2 & 12 & 5 & - & - & 5 & 17 & $27 \%$ \\
\hline
\end{tabular}

dysplasia in buccal mucosa, commisure and tongue as shown in table 2.

\section{Correlation of grade of dysplasia with the clinical type of leukoplakia}

Table 2 Correlation of Grade of Dysplasia with Site of Leukoplakia

\begin{tabular}{lccc}
\hline & \multicolumn{3}{c}{ Grade of dysplasia } \\
\hline Site of lesions (No.) & Mild & Moderate & Severe \\
Buccal mucosa (22) & 5 & 1 & 2 \\
Commisure (20) & 3 & 2 & - \\
Tongue (18) & 4 & - & - \\
Palate (1) & - & - & - \\
\hline
\end{tabular}

The different types of leukoplakia and the grade of dysplasia found within these groups is shown in table 3 and picture 1,2 .

\section{Histological criteria in oral epithelial dysplasia}

The Incidence of histological features (WHO criteria) in 27 cases diagnosed as oral epithelial dysplasia is shown in table 4.

\section{Spectrum of histopathological appearance}

Of the 61cases of oral keratotic white lesions, 35\% were benign keratosis and different spectrum of histological features as shown in table 5 and picture 3-11. 
Table 3 Correlation of Grade of Dysplasia with the Clinical Type of Leukoplakia

\begin{tabular}{lcccc}
\hline & Leukoplakia & \multicolumn{3}{c}{ Grade of dysplasia } \\
\hline Type of leukoplakia & Cases & Mild & Moderate & Severe \\
Homogenous & 54 & 11 & 1 & 0 \\
Speckled & 4 & 1 & 1 & 1 \\
Verrucous & 3 & 0 & 1 & 1 \\
\hline Total & $\mathbf{6 1}$ & $\mathbf{1 2}$ & $\mathbf{3}$ & $\mathbf{2}$ \\
\hline
\end{tabular}

\section{Predisposing factors associated with personal habits}

The distribution of different personal habits such as betel usage, tobacco smoking, chewing habits and alcohol

\section{Table 4 Histological features in 27 cases}

\begin{tabular}{lcc}
\hline \multicolumn{1}{c}{ Histological Criteria } & No of cases & Percentage \\
\hline Irregular epithelial stratification & 17 & $63 \%$ \\
Hyperplasia of the basal layer & 15 & $56 \%$ \\
Drop- shaped rete pegs & 10 & $37 \%$ \\
Increased number of mitotic figures & 8 & $29 \%$ \\
Loss of polarity of the basal cells & 17 & $63 \%$ \\
Increased nuclear- cytoplasmic ratio & 20 & $74 \%$ \\
Nuclear pleomorphism & 21 & $78 \%$ \\
Nuclear hyperchromatism & 10 & $37 \%$ \\
Enlarged nucleoli & 12 & $44 \%$ \\
Keratinization of single cells or cell groups in the & 11 & $41 \%$ \\
prickle layer & 16 & $59 \%$ \\
Loss of intercellular adherence & & \\
\hline
\end{tabular}

were presents in 15 patients (25\%) as shown in table 6 . There were 3 cases of carcinoma present in the study, 1 each of verrucous, microinvasive and invasive carcinoma which clinically presented as verrucous leukoplakia, homogenous leukoplakia and speckled leukoplakia with Table 5 Spectrum of Histopathological evidence on biopsy

\begin{tabular}{lcc}
\hline $\begin{array}{l}\text { Spectrum of Histopathologic } \\
\text { diagnosis on biopsy }\end{array}$ & No. of cases & Percent \\
\hline Leukoplakia- Benign keratosis & 22 & $35 \%$ \\
Leukoplakia- Mild dysplasia & 12 & $19 \%$ \\
Leukoplakia- Moderate dysplasia & 3 & $5 \%$ \\
Leukoplakia- Severe dysplasia & 2 & $3 \%$ \\
Verrucous hyperplasia & 1 & $2 \%$ \\
Proliferative verrucous hyperplasia & 1 & $2 \%$ \\
Leukoedema & 1 & $2 \%$ \\
Lichen keratosis & 4 & $7 \%$ \\
Lichenoid dysplasia & 3 & $5 \%$ \\
Chronic hyperplastic candidiasis & 6 & $9 \%$ \\
Lichen planus & 3 & $5 \%$ \\
Verrucous carcinoma & 1 & $2 \%$ \\
Squamous cell carcinoma (micro- & & \\
invasive) & 1 & $2 \%$ \\
Invasive squamous cell carcinoma & 1 & \\
\hline
\end{tabular}

ulceration respectively (Table 7). But due to unavailability of detail personal habits from all cases, the association between the predisposing risk factors and degree of dysplasia/ malignancy could not be drawn.

\section{Clinical site and appearance of carcinoma}

In the study clinical site of buccal mucosa 5\% (3/61) carcinoma were found in which 2 cases carcinoma in male patients and one case of carcinoma in female as depicted in table Pic- ture 2 Speckled leukoplakia: showing a non homogenous appearance

\section{Discussions:}

Oral keratotic white lesions reflect many different diseases and pathological changes. When clinical leukoplakia lesions

\section{Table 6 Distribution of Personal habits}

\begin{tabular}{lccc}
\hline \multicolumn{1}{c}{ Distribution of Personal Habits } & Male & Female & No. of cases \\
\hline Betel usage & 4 & - & 4 \\
Tobacco Smoking & 3 & - & 3 \\
Alcohol consumption & - & - & - \\
Betel usage and Tobacco smoking & 3 & 1 & 4 \\
Tobacco chewing & 2 & - & 2 \\
Tobacco smoking and alcohol & & & \\
consumption & 1 & - & 1 \\
Betel, tobacco and alcohol use & 1 & - & 1 \\
\hline Total & 14 & 1 & 15 \\
\hline
\end{tabular}

are studied microscopically, they seem to embrace a spectrum of histologic changes that may range from only increased keratosis to invasive SCC. These differences cannot be identified clinically hence a biopsy is required to establish specific diagnosis. Some of these white lesions have a potential for malignant transformation, but it is also apparent

Table 7 Age, Sex, Site \& Clinical appearance of carcinoma

\begin{tabular}{lcccl}
\hline \multicolumn{1}{c}{ Type of carcinoma } & Age & Sex & \multicolumn{1}{c}{ Site } & Clinical Appearance \\
\hline Verrucous carcinoma & 66 & $\mathrm{~F}$ & Buccal mucosa & Verrucous \\
$\begin{array}{l}\text { Squamous cell carcinoma } \\
\text { (micro-invasive) }\end{array}$ & 54 & $\mathrm{M}$ & Buccal mucosa & Speckled, erosive \\
$\begin{array}{l}\text { Invasive squamous cell } \\
\text { carcinoma }\end{array}$ & 59 & $\mathrm{M}$ & Buccal mucosa & Speckled \\
\hline
\end{tabular}

that as many as $50 \%$ of oral squamous cell carcinomas can arise from these white lesions. Therefore, biopsy with microscopic evaluation is the gold standard for the assessment of oral white lesions. Oral epithelial dysplasia is the histologic marker of premalignancy and as such it is predictive of an increased rate of development of squamous cell carcinoma ${ }^{1}$.

The term leukoplakia is employed in a clinical sense, meaning a white patch on the mucous membrane that cannot be removed by rubbing and is not classifiable as any other diagnosable disease. Leukoplakia is a common lesion and represents $6.2 \%$ of all oral biopsy specimens. Most lesions occur between 40 and 70 years of age and more commonly in men $^{3,4}$. The mean age at diagnosis of oral premalignancy is $50-69$; less than $5 \%$ of diagnoses are in patients under 30 years of age ${ }^{5}$. In our study, 40 cases (66\%) were males while 21 cases (34\%) were females. Age distribution ranged from 29 to 86 years and average age of males was 50 years while the average age of females was 54 years. Most lesions occurred between 31 and 70 years of age and more commonly in men.

In a study Neville et al reported the most common sites as buccal mucosa, alveolar mucosa and lip ${ }^{3}$ while Silverman et al also found the buccal mucosa (46\% patients) to be the most common site followed by gingiva ( $40 \%)$, palate $(27 \%)$, tongue (26\%), floor (22\%) and lip (11\%) . In our study, the 


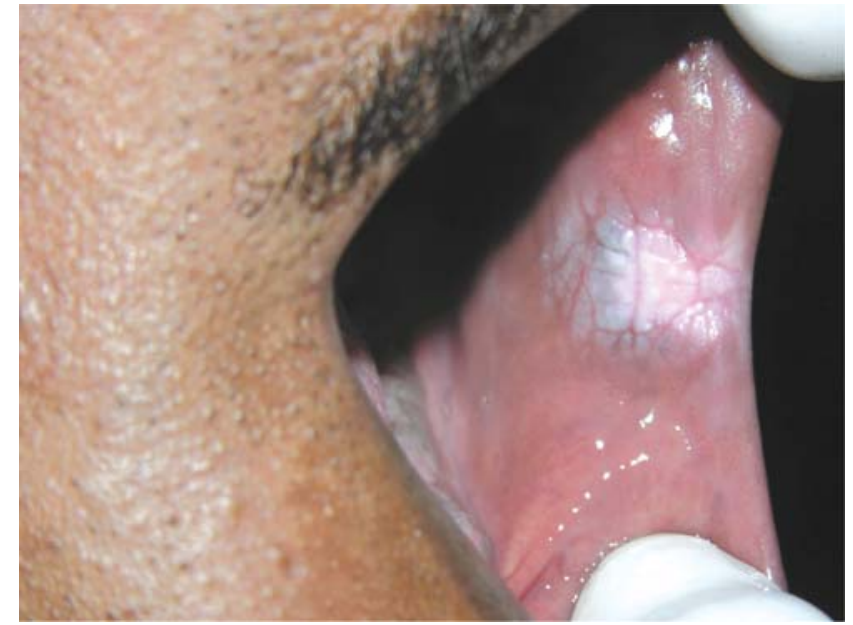

Pic. 1: Homogenous leukoplakia: showing an irregular white, homogenous plaque on the lateral buccal mucosa.

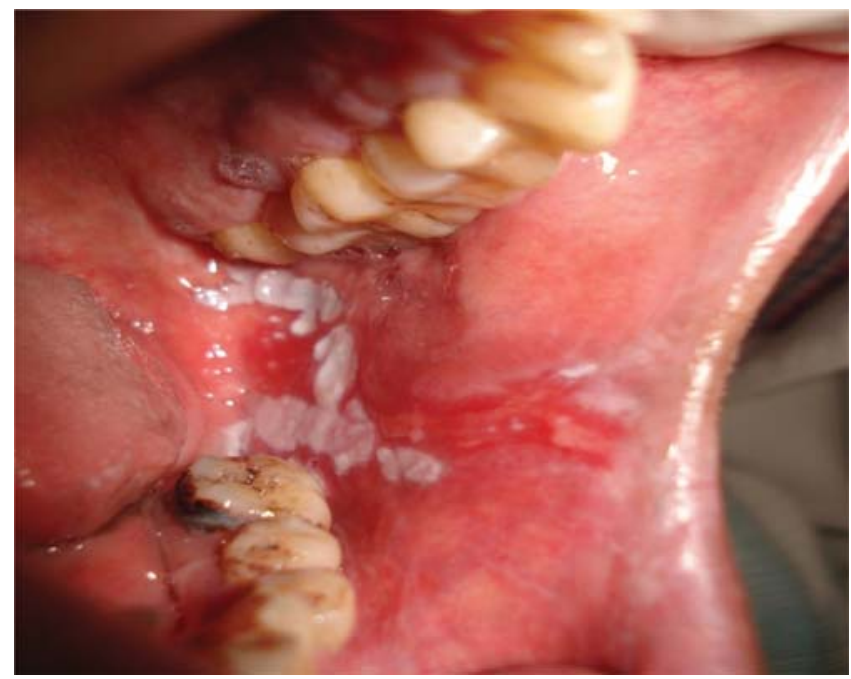

Pic. 2: Speckled leukoplakia: showing a non homogenous appearance.

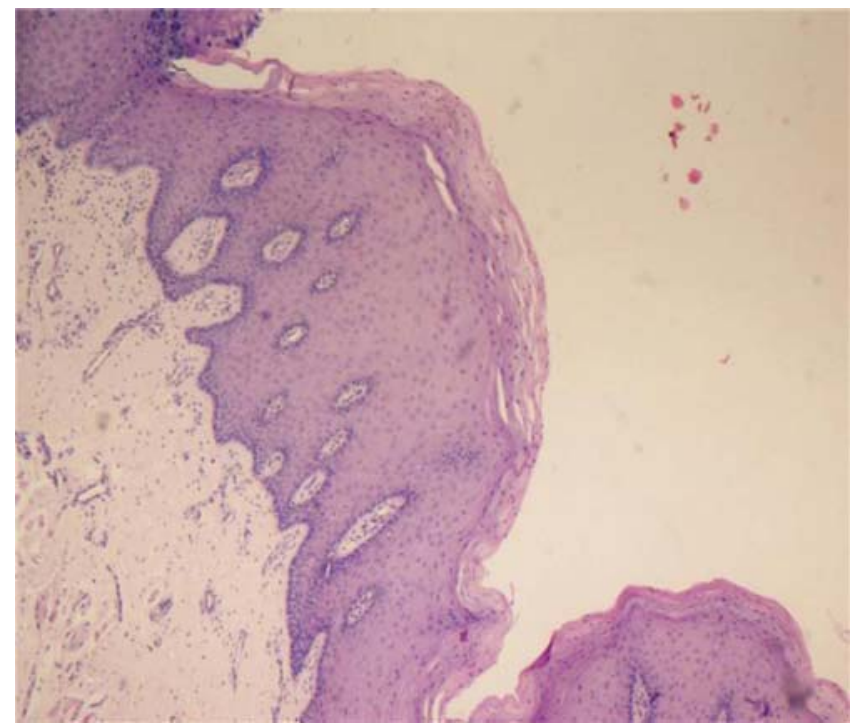

Pic. 3: Benign Keratosis: showing thickening of squamous epithelium; hyperkeratosis; no dysplasia. H\&E: 200X.

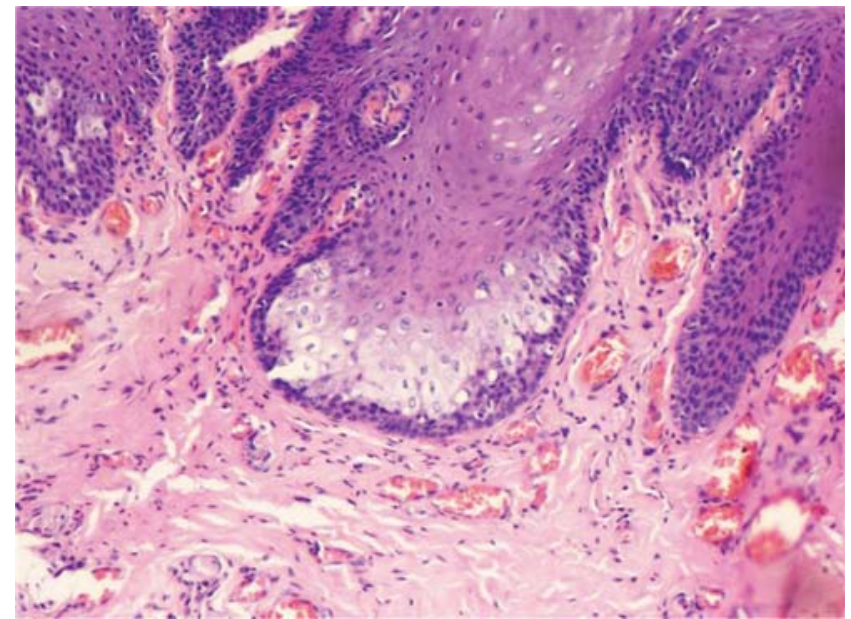

Pic. 4: Leukoedema: showing intracellular edema with abundant clear cytoplasm.

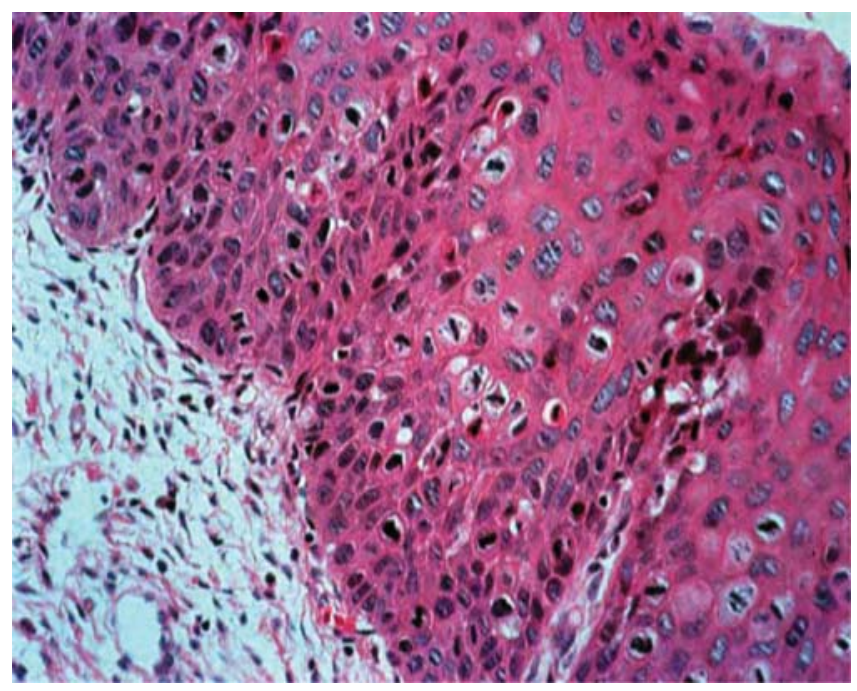

Pic. 5: Severe dysplasia: showing full thickness of the epithelium with nuclear pleomorphism, hyperchromasia \& disorderly maturation. H\&E: 400X.

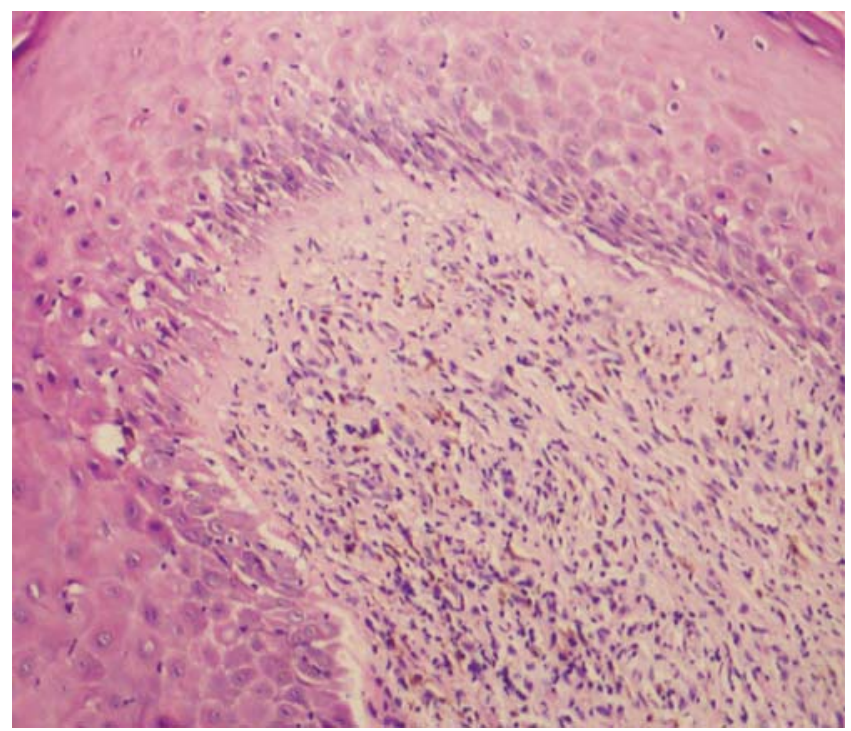

Pic. 6: Lichen planus: showing basal cell degeneration \& lichenoid infiltrate. H\&E: 400X. 


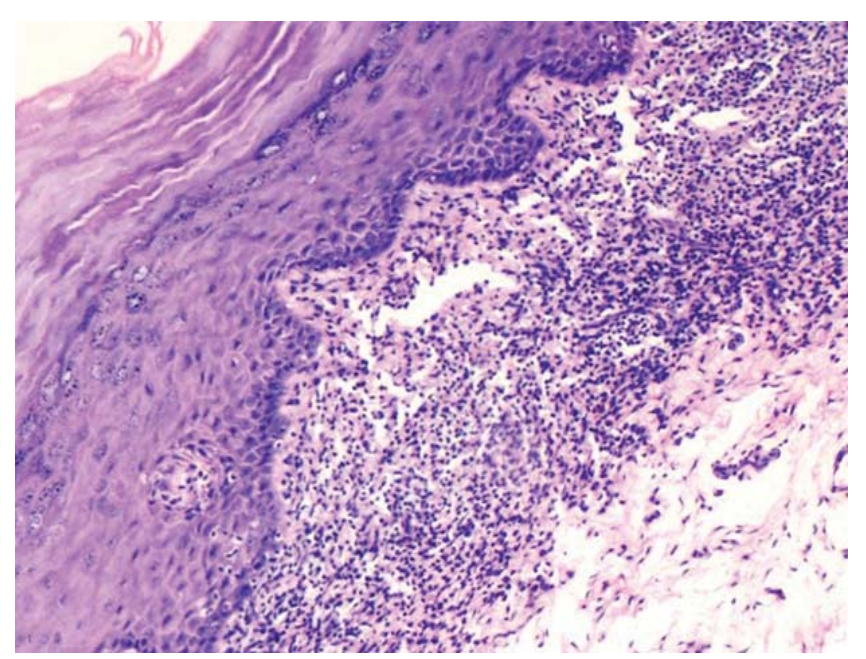

Pic. 7: Lichenoid keratosis: showing acanthosis, hyperkeratosis \& diffuse lichenoid infiltrate. H\&E: 200X.

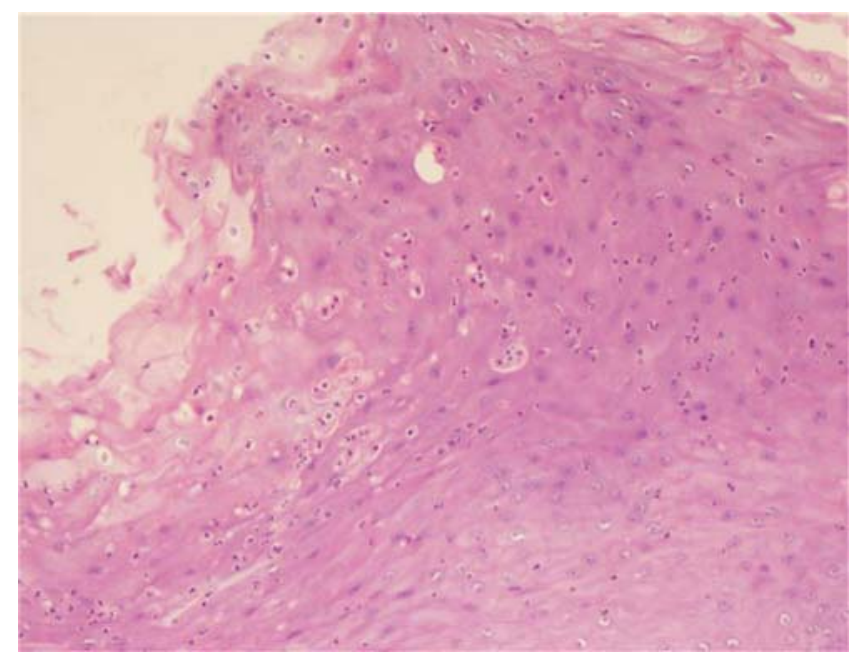

Pic. 9: Chronic hyperplastic candidiasis: showing hyperplastic epithelium, hyperkeratosis \& neutrophilic infiltrate. H\&E: 100X.

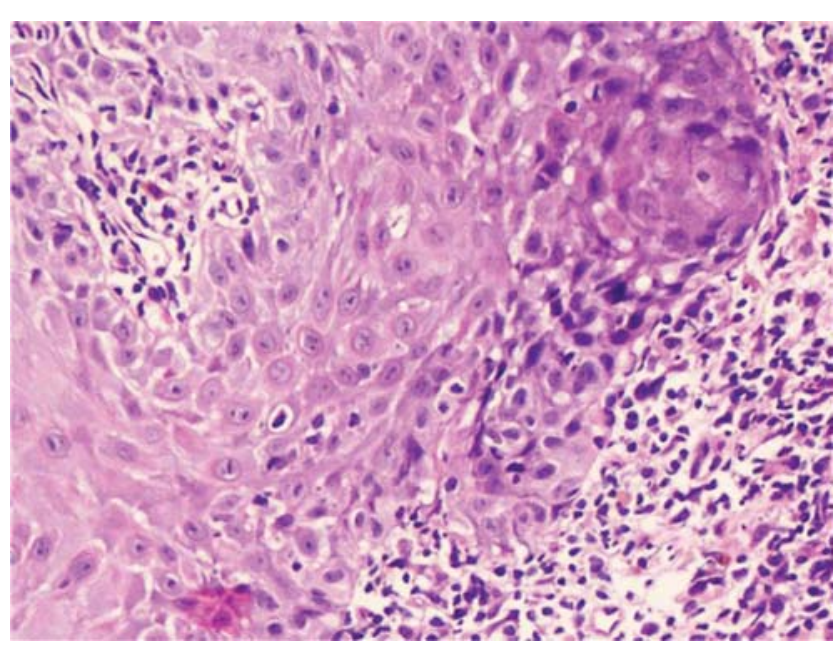

Pic. 11: Squamous cell carcinoma: showing micro-invasion. H\&E 400X.

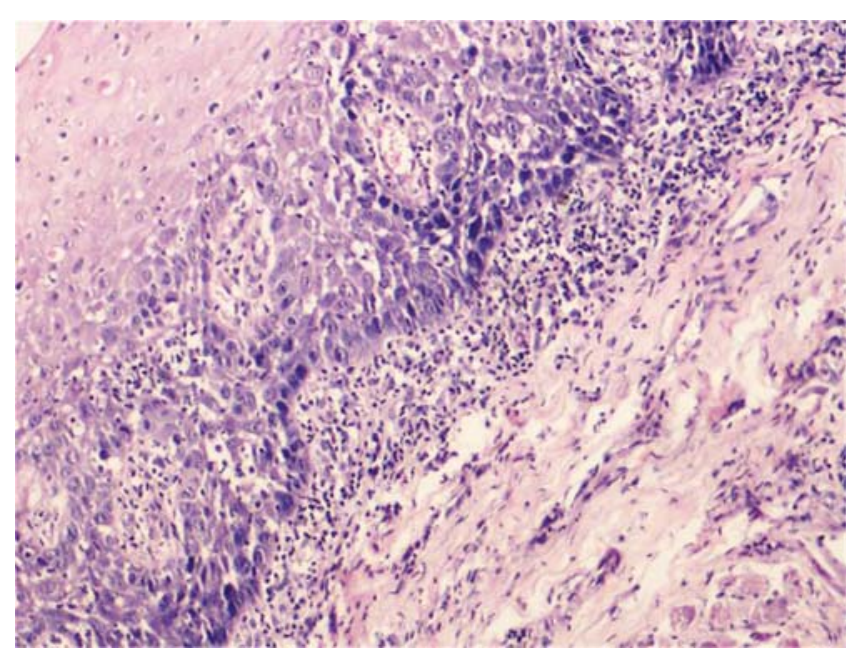

Pic. 8: Lichenoid dysplasia: showing dysplasia of basal layers \& lichenoid infiltrate. H\&E: 200X.

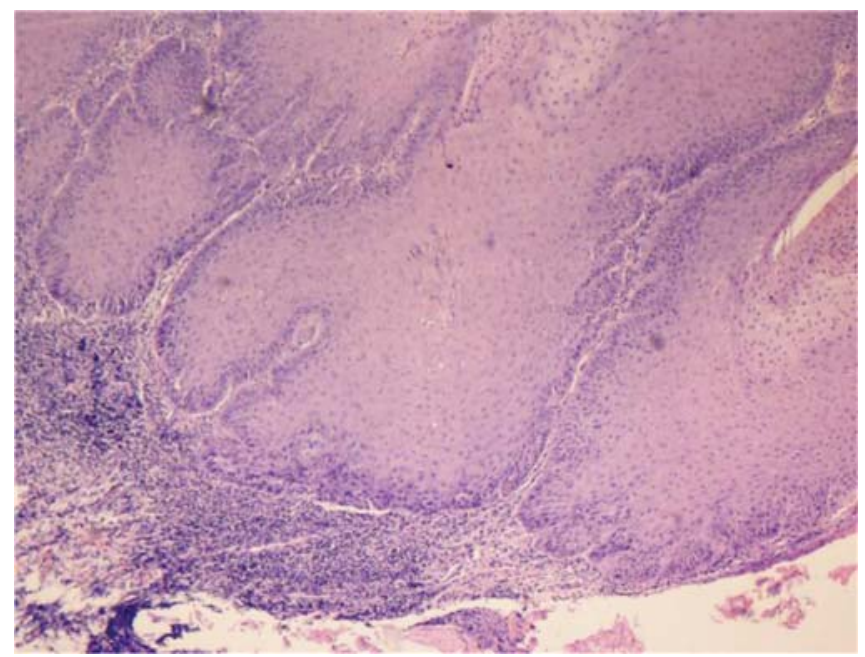

Pic. 10: Verrucous carcinoma: showing downward proliferation with bulbous rete pegs infiltrating the subepithelium H\&E: 200X.

buccal mucosa was the most common site of lesion occurring in $35 \%$ of patients, followed by commissures (33\%), tongue (30\%) and palate (2\%).

Various types of irritants have been associated with the development of hyperkeratotic oral lesions. Tobacco has been established as a significant risk factor ithe development of oral cancer with studies indicating that over $90 \%$ of oral cancer patients use tobacco. Silverman et al in their study found that while $73 \%$ of their entire study group used tobacco only $47 \%$ of those developing cancer smoked. Candidiasis has also been cited as a potential risk factor or at least a complication of leukoplakia. Distribution of personal habits in our study is given in Table 12.15 cases had some personal habits while the rest 46 cases did not have personal habits. Betel usage and both betel and tobacco use were the 
most common. There were 8/15 cases (53\%) which showed dysplasia. Clinical appearance of leukoplakia indicates a possible relationship with the probability of dysplastic or malignant findings within the lesion. As the lesion increases in thickness, the probability of dysplasia or malignancy increases. Papillary verrucous leukoplakia has the greatest chance of dysplasia/ malignancy compared with thick leukoplakia, which in turn has a higher dysplastic/ malignant potential than the thin lesion. Dysplasia is rarely seen in homogenous leukoplakia while the non-homogenous leukoplakias are almost always the seat of either dysplasia, carcinoma in situ, or squamous cell carcinoma. An erythematous or red component has been recognized as increasing the possibility of present or future dysplastic or malignant epithelial changes. Roed ${ }^{6}$ reported a seven-fold risk for malignant transformation in individuals with an erythematous component to their leukoplakias. His group showed a $1.3 \%$ transformation rate in homogenous leukoplakia versus a 9.1\% rate in red and white appearing lesions. Wood ${ }^{7}$ has reported malignant change in leukoplakia to range between $3 \%$ and $7 \%$. In our study the clinical appearances ranged from homogenous, verrucous and speckled. Among 12 of the 54 cases (22\%) of homogenous leukoplakia displayed dysplasia, of which 11 cases (92\%) showed mild dysplasia and 1 case $(8 \%)$ showed moderate dysplasia. Of the 4 cases of speckled leukoplakia, 3 cases (75\%) showed dysplasia, 1 case (25\%) each of mild, moderate and severe dysplasia. 2 of the 3 cases (66\%) of verrucous leukoplakia displayed dysplasia, of which 1 case (33\%) showed moderate dysplasia and 1 case (33\%) showed severe dysplasia.

Benign keratosis clinically present as oral keratotic white lesions. It is related to chronic rubbing or friction or an irritant against an oral mucosa and results in a presumably protective hyperkeratotic white lesion that is analogous to a callus on the skin. It commonly occurs on the lips, lateral margins of the tongue and buccal mucosa. In our study, benign keratosis with epithelial hyperplasia formed the largest group and consisted of 35\%. Silverman and colleagues monitored 257 patients with oral leukoplakia of which 235 cases showed hyperkeratosis ${ }^{8}$. Leukoplakias may be histologically divided into two main categories: those that show no atypia (dysplasia) and those that show different degrees of atypia. In 1976, Banoczy and Csiba ${ }^{9}$ in their study reported that $24 \%$ of 500 oral leukoplakias showed dysplasia while Silverman et al. as part of a larger study ${ }^{8}$ reported that $36 \%$ of 22 oral leukoplakias exhibited dysplasia. Mehta and associates ${ }^{10}$, in their study found epithelial dysplasia in $10.7 \%$ of their cases. Pindborg and Roed-Petersen ${ }^{6}$ noted a $15.4 \%$ incidence of dysplasia while Waldron and Shafer reported that in their study of 3256 cases of oral leukoplakia, 19.9\% had some degree of epithelial dysplasia. In a previous study by Banoczy and Csiba, dysplasia was observed in 28.8\%. In our study 17 (27\%) of 61 cases showed dysplasia. Of the $27 \%$ dysplastic cases, $19 \%$ were mild dysplasia, $5 \%$ moderate and 3\% severe dysplasia. 11 cases (40.7\%) from 54 homogenous leukoplakia had mild dysplasia while 1 case (3.7\%) had moderate dysplasia. 3 cases of speckled leukoplakia showed mild, moderate and severe dysplasia respectively, while 2 cases of verrucous leukoplakia showed moderate and severe dysplasia. 8 cases (47\%) of dysplasia was located in the buccal mucosa of which 5 cases were of mild, 1 moderate, and 2 severe grades. 5 cases (29\%) of dysplasia was located in the commisures of which 3 cases were of mild grade and 2 cases were of moderate grade. 4 cases (24\%) of dysplasia was located in the tongue and all showed mild grade of dysplasia.

Defining "epithelial dysplasia" as an entity with histologic abnormalities suggests that the lesion has a greater probability of undergoing malignant change than do normal tissue. The histologic grade reflects the degree of involvement: mild cases of epithelial dysplasia are those in which changes are seen within the lower third of the epithelium; moderate cases, those in which at least half the epithelium is involved; and severe cases, those in which most of the epithelium is affected. The most frequent histomorphological features seen in our study were nuclear pleomorphism in 21 out of 27 cases (78\%), increased nuclearcytoplasmic ratio in 20 cases (74\%), irregular epithelial stratification 17 cases (63\%), and 17 cases (63\%) with loss of polarity of the basal cells. In a study by Banoczy et al. ${ }^{9}$ they noted among 120 cases of dysplasia nuclear pleomorphism in $15 \%$ cases, increased nuclear-cytoplasmic ratio in 13.3\% cases, irregular epithelial stratification in $75 \%$ cases and loss of polarity of the basal cells in $23.3 \%$ cases. The correlation of the grade of dysplasia with age and sex distribution is shown in Table 1 . There were 12 cases( $71 \%$ ) of dysplasia in the males and 5 cases (28\%) of dysplasia in the females from a total of 17 cases of dysplasia. Among the males, mild dysplasia was seen in 7 cases (58\%) of the 12 cases, and most cases were observed between the ages of 41 and 50 years. There were 3 cases (25\%) of the 12 cases of moderate dysplasia among the men between the ages 31 and 60 years, and there were 2 cases (16\%) of severe dysplasia between the ages of 41 and 60 years. Among the females, there were 5 (100\%) out of 5 cases of mild dysplasia. 1 case between the ages of 41 and 50 years, and 4 cases between the ages of 51 and 70 years.

Proliferative verrucous leukoplakia is a distinct clinical form of oral leukoplakia defined by its progressive clinical course, changing clinical and histopathological features and a potential to develop into cancer. It behaves in a more aggressive and relentless manner than the more innocuous white oral lesions that it can resemble clinically.

Verrucous Hyperplasia is histologically difficult to distinguish from verrucous carcinoma at its early form, although the characteristic feature of a blunted epithelial front pushing into underlying lamina propria seen in verrucous carcinoma is absent in verrucous hyperplasia. In the current study there was 1 case of verrucous hyperplasia. Lichenoid dysplasia is fundamentally a precancerous 
process with lichenoid features. There were 3 cases of lichenoid keratosis (5\%) with dense lichenoid infiltrate in the dermis and keratosis, and no evidence of basal cell degeneration to suggest lichen planus; no epithelial dysplasia was seen.

Chronic hyperplastic candidiasis most commonly presents as a white plaque that cannot be rubbed off and is most frequently found in the commissural regions of the oral mucosa ${ }^{11}$. In as much as candidal organisms are found in up to $82 \%$ of leukoplakias, the question arises about whether specific cases are primary or secondary candidiasis. According to several reports, the leukoplakia that harbour candidal hyphae appear to constitute from $7 \%$ to $50 \%$ of all leukoplakias. In our study there were $9 \%$ cases of candidiasis.

Oral cancers in their early stages can simulate benign or premalignant lesions. In a study done by Banoczy et $\mathrm{al}^{12}$ in 500 histologic sections clinically diagnosed as leukoplakia, 48 cases $(9.6 \%)$ showed carcinoma. Silverman et al in their study found $7 \%$ carcinoma. A previous study done in Manipal Health System Hospital ${ }^{13}$ found prevalence of oral malignancies at $1.7 \%$. Males (2.14\%) had a higher predilection for this lesion than females $(1.12 \%)$ and it was observed more often in the age group of 41-60 years. Main site of involvement was the buccal mucosa and the lateral border of the tongue. All carcinomas were located in the buccal mucosa. Clinical diagnosis for the case of verrucous carcinoma was verrucous leukoplakia, while the cases with histologic diagnosis of micro-invasive and invasive SCC appeared as speckled leukoplakia and the invasive carcinoma showed ulceration.

\section{CONCLUSION}

The prevalence of oral keratotic white lesions, their clinical appearance correlation to the histopathological findings was higher in males when compared to females in the ratio of 2:1.

Oral keratotic white lesions demonstrated wide spectrum of histopathological features ranging from benign keratosis to varying grades of dysplasia to invasive squamous cell carcinoma to specific diagnosis like lichen planus, candidiasis.

Predisposing factors like tobacco smoking, betel usage, alcohol were present in 15 patients. $46 \%$ of the 15 patients showed different degrees of dysplasia to carcinoma. Due to unavailability of detailed personal habits from all cases, the association between predisposing risk factors and transformation of malignancy could not be drawn. Further study is require to conclude the association between predisposing risk factors and transformation of malignancy.

\section{LIMITATIONS OF THE STUDY}

Being a retrospective study, some of the clinical findings like the size of the lesion etc. could not be ascertained.

Details of personal habits were not available for all cases; hence an association between the predisposing risk factors and degree of dysplasia / malignancy could not be drawn from this study.

\section{REFERENCES}

1. Lumerman, H., Freedman, P., Kerpel, S. 1995. Oral epithelial dysplasia and the development of invasive squamous cell carcinoma. Oral Surg. 79: 321-329.

2. Barnes, L., Eveson, JW., Reichart, P., Sidransky, D. 2005. WHO classification of tumours. Pathology and genetics of head and neck tumours. Lyon.

3. Neville Brad, W., Day Terry, A. 2002. Oral cancer and Precancerous Lesions. CA: A Cancer Journal for Clinicians. 52: $195-215$.

4. Reddi Sanjay P., Shafer Adam, T. 2006. Oral Premalignant lesions: Management considerations. Oral and Maxillofacial Surg Clin N Am. 18: 425-568.

5. Jr. Silverman S., Gorsky, M., Lozada-Nur, F., Giannotti, K.A. 1991. prospective study of findings and management in 214 patients with oral lichen planus. Oral Surg Oral Med Oral Pathol. 72: 665-70.

6. Roed Petersen, B. 1971. Cancer development in Oral Leukoplakia. J e Dent Res. 50: 711.

7. Norman, K., Wood., Goaz., Paul, W. 1997. Differential diagnosis of Oral and Maxillofacial lesions.

8. Silverman S, Jr., Gorsky, M., Lozada-Nur, F. 1984. Oral leukoplakia and malignant transformation. A follow-up study of 257 patients. Cancer. 53: 563-568.

9. Banoczy, J., Csiba, A. 1976. Occurrence of epithelial dysplasia in oral leukoplakia: analysis and follow-up study of 120 cases. Oral Surg Oral Med Oral Pathol. 42: 766-74.

10. Mehta, FS., Pindborg, JJ., Daftary, DK., Gupta, PC. 1969. Oral leukoplakia among Indian Villagers. the association with smoking habits. Br Dent J. 127: 73-77.

11. Sitheeque, MAM., LP, Samaranayake. 2003. Chronic hyperplastic candidiasis. Crit Rev Oral Med. 14(4): 253-267.

12. Banoczy, J. Sugar, L. 1972. Longitudnal Studies in Oral Leukoplakia. J Oral Pathol. 1: 265-272.

13. Mathew, AL., Pai, KM., Sholapurkar, AA., Vengal, M. 2008. The prevalence of oral mucosal lesions in patients visiting a dental school in Southern India. Indian J Dent Res. 19(2). 